\title{
BMJ Open Is movement variability altered in people with chronic non-specific low back pain: a protocol for a systematic review
}

\author{
Amal M Alsubaie, ${ }^{1,2}$ Masood Mazaheri, ${ }^{1}$ Eduardo Martinez-Valdes (D) , \\ Deborah Falla (i) ${ }^{1}$
}

To cite: Alsubaie AM, Mazaheri M, MartinezValdes $\mathrm{E}$, et al. Is movement variability altered in people with chronic non-specific low back pain: a protocol for a systematic review. BMJ Open 2021;11:e046064. doi:10.1136/ bmjopen-2020-046064

- Prepublication history and additional supplemental material for this paper are available online. To view these files, please visit the journal online (http://dx.doi.org/10.1136/ bmjopen-2020-046064).

Received 20 October 2020 Accepted 10 May 2021

D) Check for updates

(c) Author(s) (or their employer(s)) 2021. Re-use permitted under CC BY-NC. No commercial re-use. See rights and permissions. Published by BMJ.

${ }^{1}$ Centre of Precision

Rehabilitation for Spinal Pain (CPR Spine), School of Sport, Exercise and Rehabilitation

Sciences, University of Birmingham, Birmingham, UK ${ }^{2}$ Department of Physical Therapy, Faculty of Medical Rehabilitation Sciences, King Abdulaziz University, Jeddah, Saudi Arabia

Correspondence to Professor Deborah Falla; d.falla@bham.ac.uk

\section{ABSTRACT}

Introduction Motor variability is an important

feature when performing repetitive movement, and in asymptomatic people functional tasks are typically performed with variable motor patterns. However, in the presence of chronic non-specific low back pain (LBP), people often present with different motor control strategies than those without pain. Movement variability has been assessed using a wide range of variables, including kinetic and kinematic components of motion. This has resulted in a wide range of findings reported in the literature and some contradicting results. Therefore, the aim of this systematic review is to investigate whether the amount and structure of motor variability are altered in people with chronic non-specific LBP, during both repetitive nonfunctional and functional tasks.

Methods and analysis This protocol for a systematic review is informed by Cochrane guidelines and reported in line with the Preferred Reporting Items for Systematic Reviews and Meta-Analysis Protocols. MEDLINE, EMBASE, CINAHL, ZETOC, Web of Science, PubMed and Scopus will be searched from their inception to December 2020 along with a comprehensive search of grey literature and key journals. Two independent reviewers will conduct the search, extract the data, assess risk of bias (using the Downs and Black Scale) for the included studies and assess overall quality of evidence based on Grading of Recommendations, Assessment, Development and Evaluation guidelines. Meta-analysis will be conducted if deemed appropriate. Alternatively, a narrative synthesis will be conducted and evidence summarised as an increase, decrease or no change in the motor variability of people with LBP compared with healthy controls.

Ethics and dissemination This study raises no ethical issues. Results will be submitted for publication in a peer review journal and presented at conferences.

PROSPERO registration number CRD42020211580.

\section{INTRODUCTION}

The socioeconomic burden of low back pain (LBP) is increasing rapidly despite advances in diagnosis and management. In approximately $85 \%-90 \%$ of cases, the pathoanatomical cause of LBP cannot be identified, hence the term non-specific LBP.${ }^{2}$ One of the main
Strengths and limitations of this study

- This systematic review will be the first to rigorously summarise and evaluate the current body of evidence assessing motor variability in people with chronic non-specific low back pain.

- The protocol is written in accordance with the Preferred Reporting Items for Systematic Reviews and Meta-Analyses Protocols guidelines.

- The methodology of this protocol allows for a wide range of tasks to be considered, including functional and non-functional tasks.

- Based on the methodological diversity among possible eligible studies, a meta-analysis may not be possible.

- The qualitative method used to synthesise the evidence provides the direction of the effect for individual studies without considering the overall magnitude or precision of the effect size.

reasons for individuals with LBP to seek care is limitation of their functional ability ${ }^{3}$ which may include reduced physical performance (e.g., strength, mobility, balance, endurance, and coordination). ${ }^{4}$

Repetitive movements during work or activities of daily living can result in injury, especially of the low back..$^{5}$ Variation in movements, posture or muscle activity has been suggested as an effective strategy to minimise the load associated with repetitive movements and hence may prevent or delay any potential musculoskeletal injury. ${ }^{6}$ Motor variability is an important feature to consider when performing repetitive movement, and in asymptomatic people functional tasks are typically performed with variable motor patterns. ${ }^{7}$ The biological systems illustrate an inherited normal variation (lying between too much variability and complete repeatability) in both space and time to maintain or achieve functional skills. ${ }^{8}$ However, in the presence of LBP, people often present with 
different motor control strategies than those without pain, ${ }^{9}$ and changes in motor variability are often reported in both kinematic parameters (e.g., reduced trunk-pelvis coordination and the degrees of freedom during walking or other activities of daily living $)^{1011}$ and neuromuscular variables (e.g., reduced variability of muscle activity during repetitive lifting or other fatiguing tasks). ${ }^{6} 12$

Using different methods to measure human movement, variability has been assessed at various levels, including kinetic and kinematic components of motion, coordinative aspects of movement, and muscle activity and patterns of muscle recruitment. ${ }^{13}$ Recently, with the emergence of a range of different mathematical approaches, researchers have used linear and non-linear tools to analyse the amount and structure of motor variability, respectively. ${ }^{81415}$ Linear tools to measure variability in terms of the statistical variance (e.g., range, standard deviation and coefficient variation) provide information only about the quantity of the movement but give no insight regarding the temporal variations of movement. ${ }^{8}$ Therefore, non-linear tools are used to reveal the structure of variability or the time-evolving nature of the variations and hence provide additional information about the level of complexity of the motor system. ${ }^{15}{ }^{16}$ Nonlinear measures employ a wide range of mathematical tools to capture the structure of variability, such as largest Lyapunov exponent, ${ }^{16}$ uncontrolled manifold method or the noise-tolerance-covariance decomposition, ${ }^{14}$ entropy measures, recurrence quantification analysis, discrete data analysis, normalised root mean square, Cauchy criterion and cluster analysis. ${ }^{15}$

The various methods used to measure variability in people with LBP have resulted in a wide range of results with some contradicting findings. For example, some studies report increased movement variability during gait in people with LBP. ${ }^{1718}$ Other studies report reduced variability during functional or non-functional tasks, which supports the argument that people with LBP increase trunk stiffness as a protective response to pain. ${ }^{19}{ }^{20}$ Other studies have shown no significant difference or change in motor variability in people with LBP. ${ }^{21} 22$ The inconsistency in the literature regarding the influence of LBP on motor variability highlights the need to systematically review and synthesise the current literature. To date, there is no comprehensive systematic review that assesses motor variability in people with chronic non-specific LBP. There are reviews that examined motor control changes in general, however, in this case, only specific functional tasks such as gait or standing were considered, ${ }^{23}{ }^{24}$ or the focus was on an older population only. ${ }^{25}$

Therefore, the aim of this systematic review is to investigate whether the amount and structure of motor variability measured in the thoracolumbar and lumbopelvic regions are altered in people with chronic non-specific LBP. Multiple outcome measures are considered, including kinetic, kinematic, coordination and spatiotemporal parameters, during both repetitive non-functional and functional tasks.
Table 1 Summary of inclusion and exclusion criteria

\begin{tabular}{ll}
\hline Inclusion criteria & \\
\hline Population & $\begin{array}{l}\text { Adults ( } \geq 18 \text { years old), men and women } \\
\text { with chronic non-specific LBP and } \\
\text { healthy controls }\end{array}$ \\
\hline $\begin{array}{l}\text { Indicator/ } \\
\text { exposure }\end{array}$ & $\begin{array}{l}\text { Motion analysis systems (eg, } \\
\text { optoelectronic systems, inertial } \\
\text { measurement unit sensors) }\end{array}$ \\
\hline Comparison & $\begin{array}{l}\text { Difference between people with chronic } \\
\text { non-specific LBP and healthy controls }\end{array}$ \\
Outcomes & $\begin{array}{l}\text { Amount and/or structure of movement } \\
\text { variability based on linear or non-linear } \\
\text { measures }\end{array}$ \\
Study type & $\begin{array}{l}\text { Quantitative cross-sectional } \\
\text { observational studies }\end{array}$ \\
\hline
\end{tabular}

\section{Exclusion criteria}

\begin{tabular}{ll} 
Population & $\begin{array}{l}\text { Individuals under the age of 18, people } \\
\text { with LBP attributable to a specific } \\
\text { pathology, concurrent systemic } \\
\text { disorders, surgery, cardiovascular } \\
\text { conditions or pregnancy }\end{array}$ \\
$\begin{array}{ll}\text { Indicator/ } \\
\text { exposure }\end{array}$ & None \\
Comparison & None \\
Outcomes & $\begin{array}{l}\text { Studies used spatiotemporal parameters } \\
\text { based on neuromuscular variables }\end{array}$ \\
Study type & $\begin{array}{l}\text { Cadaveric or animal studies, single- } \\
\text { subject case reports and longitudinal } \\
\text { cohort studies. Studies not written in } \\
\text { English will be excluded }\end{array}$ \\
\hline
\end{tabular}

LBP, low back pain.

\section{METHODS}

The protocol for this review was developed in accordance with the updated guidelines of the Cochrane Back and Neck Group, Cochrane Handbook for Systematic Reviews of Interventions and the Preferred Reporting Items for Systematic Reviews and Meta-Analyses Protocols (PRISMA-P) ${ }^{26-28}$ (online supplemental file 1). This protocol was registered on PROSPERO on 10 December 2020.

\section{Eligibility criteria}

The inclusion and exclusion criteria of the studies to be included in the review are detailed using the PICOS (P: Population; I: Indicator/Intervention; C: Comparator; O: Outcome(s); S: Study design) framework ${ }^{28}{ }^{29}$ (table 1).

\section{Population}

Studies that investigated adults aged 18 years or older with chronic non-specific LBP that persisted for at least 3 months with no diagnosable underlying pathology will be considered for inclusion. ${ }^{30}$ For the purpose of this review, adults without a history of LBP will represent the control group. Studies will be excluded if they recruited people with LBP due to trauma, fractures, spinal stenosis or radicular pain. Studies will be eligible that included people 


\section{Outcome measures domains}

\begin{tabular}{|c|c|c|c|}
\hline Concept measured & Broad domains & \multicolumn{2}{|c|}{ Narrow domains } \\
\hline \multirow{8}{*}{$\begin{array}{l}\text { Movement variability, captured using } \\
\text { movement outcomes specifically } \\
\text { related to thoracolumbar and } \\
\text { lumbopelvic segments during both } \\
\text { functional and non-functional tasks }\end{array}$} & \multirow{2}{*}{$\begin{array}{l}\text { Amount or magnitude of } \\
\text { variability - for example, using } \\
\text { traditional linear measures } \\
\text { (statistical variance) }\end{array}$} & \multicolumn{2}{|l|}{ SD } \\
\hline & & \multicolumn{2}{|l|}{ Range } \\
\hline & \multirow{6}{*}{$\begin{array}{l}\text { Structure and nature of } \\
\text { variability - for example, using } \\
\text { non-linear dynamics }\end{array}$} & \multirow[t]{2}{*}{ Intra-trial } & Entropy measure \\
\hline & & & Recurrence quantification analysis \\
\hline & & \multirow{4}{*}{$\begin{array}{l}\text { Inter-trial } \\
\text { Inter-subject }\end{array}$} & Normalised root mean square \\
\hline & & & Cauchy criterion \\
\hline & & & Cluster analysis \\
\hline & & & Uncontrolled manifold \\
\hline
\end{tabular}

with LBP and healthy participants with no concurrent systemic disorders, including rheumatic and neuromuscular disorders, spinal deformity or surgery, cardiovascular conditions and pregnancy.

\section{Indicator}

Studies will be included if they used any method to quantify spinal movement at the thoracolumbar and lumbopelvic level in the form of kinetic, kinematic, coordination or spatiotemporal parameters using motion analysis systems (eg, optoelectronic systems, inertial measurement unit sensors). ${ }^{19} 31$

\section{Comparison}

Included studies should have compared motor variability between individuals with chronic non-specific LBP and healthy controls while preforming functional (eg, gait) or non-functional tasks (eg, repetitive trunk movements).

\section{Outcomes}

Studies that used any outcome to quantify motor variability will be considered. The outcomes include the amount of motor variability captured by linear tools or the structure of motor variability recorded by non-linear tools. ${ }^{8}$ The wide range of movement variables may use kinetic or kinematic components of motion, coordinative aspects of movement or spatiotemporal parameters (table 2).

\section{Study design}

Observational cross-sectional studies will be reviewed, excluding cadaveric or animal studies and single-subject case reports as well as studies not written in English.

\section{Information sources}

The following databases will be searched from their inception to December 2020: MEDLINE (OVID Interface), EMBASE (OVID Interface), CINAHL (EBSCO Interface), ZETOC (EBSCO Interface), Web of Science, PubMed and Scopus. In addition to the database searching, hand-searching of key journals will be conducted. This will include the following key journals:
Journal of Electromyography and Kinesiology, Clinical Biomechanics, Journal of Biomechanics, Human Movement Science, The Clinical Journal of Pain, Spine, Journal of Orthopaedic $\mathcal{E}$ Sports Physical Therapy, Musculoskeletal Science and Practice and Journal of Back $\mathcal{E}$ Musculoskeletal Rehabilitation.

Distinguished authors in the field will be contacted to identify any relevant work which is currently in preparation or unpublished to carry as extensive search as possible to reduce the risk of publication bias and to identify as much relevant evidence as possible. Grey literature will be included in the search using the British National Bibliography for Report Literature, OpenGrey database, ProQuest Dissertations \& Theses Global, and EThOs. Key congresses and meetings in the field will be assessed from 2017 to 2020, including the World Congress of Biomechanics and the International Society of Electrophysiology and Kinesiology congresses. In accordance with the Methodological Expectations of Cochrane Intervention Reviews standards, hand-searching will be conducted to check reference lists in included studies and any relevant systematic reviews identified. ${ }^{32}$

\section{Search strategy}

A comprehensive, systematic and reproducible search strategy will be completed by the lead author (AMA). The search strategy has been developed using medical subject heading (MeSH) (including exploded terms) combined with keywords to ensure maximal retrieval. The search strategy will be developed in accordance with the defined PICOS components and will be linked with the Boolean terms AND/OR where relevant. There will be no restrictions implemented during the search in terms of the study design, region or date. The search strategy will remain consistent between databases. However, appropriate modifications with relevant syntax and MeSH terms will be performed to the main search strategy to adapt for other databases. A draft-developed search strategy for MEDLINE (OVID interface) database is available in online supplemental file 2 . 


\section{Data management}

Search data will be imported into EndNote V.X9 (Clarivate Analytics), including citations, abstracts and full text of relevant studies. One reviewer (AMA) will upload studies, identify any duplicate results and remove these prior to the screening process. A complete list of all potentially eligible studies with full text will be stored in EndNote V.X9 and is ready for screening process by two independent reviewers (AMA and MM).

\section{Selection process}

An electronic screening form will be created and piloted based on the eligibility criteria to subcategorise the studies into include/exclude/unclear. In the initial step of the screening process, AMA and MM will assess and subcategorise studies based on the title and abstract using the developed screening form. In the case that the eligibility is unclear from the title and abstract, the reviewers will read the full text of the article to assess inclusion. If it is still unclear, reviewers will discuss article eligibility. In the event of disagreement, a third reviewer (DF) will be asked to mediate the process. The study selection process will be summarised and depicted using the PRISMA flow diagram. ${ }^{27}$ During all assessment stages, agreement between reviewers will be estimated with percentage of agreement and the $\kappa$ statistic using SPSS for Windows statistical software package (IBM SPSS Statistics V.25).

\section{Data collection process}

Using a standardised form developed to extract data, both reviewers (AMA and MM) will independently extract information from eligible studies. Any disagreement between reviewers will be mediated through discussion with a third reviewer (DF) if needed. Prior to data collection, the data extraction form will be piloted to ensure all relevant information is collected, and any necessary changes will be implemented prior to data extraction from all eligible studies.

\section{Data items}

The characteristics of the included studies are summarised and presented in table 3. In the case of missing data or if the results are presented in an ambiguous way, the corresponding author will be contacted for clarification. If the author does not respond within a set time frame (up to 6 weeks) or the provided clarification affects the eligibility, the study will not be considered for review. If two or more papers appeared to use the same sample, the authors will be contacted for further information to ensure data are not duplicated in the review.

\section{Risk of bias}

Unlike the abundance of instruments developed to judge the methodological quality of randomised clinical trials, there is lack of a gold-standard instrument designed for quantifying the quality of observational cross-sectional studies. Hence, a modified version of Downs and Black Scale, ${ }^{33}$ designed for assessing the quality of both

\begin{tabular}{ll}
\hline $\begin{array}{l}\text { Table } 3 \text { Characteristics of included studies } \\
\text { Information } \\
\text { area }\end{array}$ & Data extracted \\
\hline $\begin{array}{l}\text { General study } \\
\text { information }\end{array}$ & $\begin{array}{l}\text { Authors } \\
\text { Year of publication } \\
\text { Title } \\
\text { Study design }\end{array}$ \\
Patient & $\begin{array}{l}\text { Age, sex, anthropomorphic data, sample } \\
\text { characteristics }\end{array}$ \\
& $\begin{array}{l}\text { size (healthy and LBP) } \\
\text { pain intensity, pain location) }\end{array}$ \\
Methodology & $\begin{array}{l}\text { Setting } \\
\text { Details of task preformed (functional } \\
\text { or non-functional, length of recording, } \\
\text { repetition, position, etc) } \\
\text { Instrument used (3D capture system, } \\
\text { Inertial measurement unit sensor etc) } \\
\text { Regions of the spine assessed (eg, } \\
\text { thoracolumbar or lumbopelvic) }\end{array}$ \\
$\begin{array}{l}\text { Amount or structure of motor variability } \\
\text { using any movement outcome related to } \\
\text { kinetic, kinematic and coordinative aspects } \\
\text { of movement or spatiotemporal parameters } \\
\text { of movement }\end{array}$ \\
\hline Outcome
\end{tabular}

3D, three-dimensional; LBP, low back pain.

randomised and non-randomised studies, will be used for quality rating in this study. The modified version consists of four domains (12 items), including quality of reporting (7 items), the generalisability of results or external validity (1 item), the relationship between LBP and outcomes, known as internal validity (4 items) and the adequacy of sample size or study power (1 item) (table 4 ). Two authors (AMA and MM) will separately assess the quality of the eligible studies.

\section{Data synthesis}

Based on the methodological diversity revealed among the possible eligible studies during the scoping search, pooling of data may not be possible; however, this will not be ruled out and meta-analysis will be conducted if possible. If a meta-analysis is deemed not possible, a narrative synthesis will be performed. An initial step of our synthesis is to systematically and comprehensively assess the results of each study-highlight any important characteristics of the studies, especially the important similarities or differences. Ideally, the processes of narrative synthesis should avoid bias by following a clear specified method in advance. We will make further decisions at review stage about how best to organise and present the data based on the actual review findings. ${ }^{34}$

Results of the included studies will be organised in subgroups in the same order as the comparisons and outcomes. To improve consistency, the following items will be contained ${ }^{26}$ : overall quality of evidence, the number of participants, the outcome measures, results 
Table 4 Quality rating instrument adjusted specifically for the current review (informed by Downs and Black Scale)

Item Scoring guideline
Reporting
1. Is the hypothesis/aim/
objective of the study clearly
described?
2. Are the main outcomes to
be measured clearly described
in the Introduction or Methods
section?

3. Are the inclusion/exclusion

of the participants included in the study clearly described?

$\begin{array}{ll}\begin{array}{l}\text { 4. Are the demographic } \\ \text { characteristics of the } \\ \text { participants included in the } \\ \text { study clearly described? }\end{array} & \begin{array}{l}\text { The question is answered 'yes' if } \\ \text { information about age and gender } \\ \text { is provided }\end{array} \\ \begin{array}{l}\text { 5. Are the clinical } \\ \text { characteristics of the }\end{array} & \begin{array}{l}\text { The question is answered 'yes' } \\ \text { if information about LBP type, } \\ \text { study clearly described? }\end{array}\end{array}$

6. Is the treatment history of the LBP participants clearly described?

7. Does the study provide estimates of the random variability in the data for the main outcomes?

\section{External validity}

8. Were the participants who were asked to participate in the study representative of the entire population from which they were recruited?

The question is answered 'yes' if studies have provided quantitative values of the SE, SD or $\mathrm{Cl}$ (for normally distributed data) and IQR (for non-normally distributed data)

Internal validity/bias and confounding

9. If any of the results of the study were based on 'data dredging', was this made clear?

The question is answered 'yes' if the studies have used several recruitment methods (eg, selfreport, hospital, insurance companies)

The question is answered 'yes' if no retrospective unplanned subgroup analyses were reported

10. Was an attempt made to blind those measuring the main outcomes?

11. Were the main outcome measures representing movement variability reliable?

The question is answered 'yes' if there is a reference to a reliability study or information on these features in the paper and this paper should cover the field of interest (LBP)

12. Were controls matched with LBP participants in important characteristics?

The question is answered 'yes' if appropriate matching on confounders, that is, age and gender, was performed or if adjustment for these variables is made in the statistical analysis

\section{Power}

13. Was there an appropriate sample size of LBP participants and controls?

The question is answered 'yes' if a sample size justification or power description is provided of quantitative analyses (effect size/ Confidence interval CI) and results of qualitative analyses (direction of the effect; an increase, decrease or no change in the motor variability of people with LBP compared with healthy controls).

Examining the effects of heterogeneity will also be assessed, especially how the results of the included studies might be affected by factors such as methodological differences between studies. ${ }^{34}$ The robustness of the results will be assessed with a sensitivity analysis to test the effect of quality/risk of bias on the results by excluding studies that were characterised as high risk of bias. ${ }^{35}$

\section{Confidence in cumulative estimate}

The Grading of Recommendations Assessment, Development and Evaluation (GRADE) method will be used to rate the overall cumulative quality of the evidence across individual studies. ${ }^{36}$ The pooled data will be assessed based on five determinants of the GRADE: risk of bias ${ }^{37}$; inconsistency, heterogeneity in studies in the review (eg, age group and severity of spinal pain $)^{38}$; indirectness; imprecision (larger sample sizes indicate greater precision) ${ }^{39}$; and, finally, publication bias arising from nonpublication of relevant results. ${ }^{40}$

As it is anticipated that the included studies will be mostly observational studies with cross-sectional designs, these studies will be given a 'LOW' rating initially in the GRADE framework. However, from this point, the quality of evidence of the study can then be downgraded or upgraded depending on the above determinants. It can also be upgraded based on the large effect sizes or a large number of studies indicating the same direction of effect. Following this process subsequently, quality of evidence will be graded as 'VERY LOW', 'LOW', 'MODERATE' or 'HIGH' according to the GRADE guidelines. ${ }^{41}$

\section{Patient and public involvement}

The research question in this study forms part of a larger discussion within our patient and public involvement meetings. Patients will not be involved in the analysis and data collection of the systematic review.

\section{Clinical implications of this study}

Motor variability is considered as an important feature of motor control and is therefore relevant to measure and consider in assessment and management of people with LBP $^{8}$ However, it is not well established to what extent motor variability can be affected in the presence of chronic LBP. Thus, the results of this systematic review may identify various features of motor variability which are altered in people with chronic LBP, and subsequently influence the direction of clinical practice.

\section{ETHICS AND DISSEMINATION}

No ethical approval is required for this review as the data that will be used in this systematic review will be collected from previously published studies. The results of this

LBP, low back pain. 
systematic review will be submitted for publication in a peer review journal and presented at conferences.

\section{Amendment protocol}

Where amendments are required from the protocol during the review process, these amendments will be documented on PROSPERO. All amendments will be clearly marked with the date of the amendment and a description of the changes, alongside a justification for the change.

Twitter Amal M Alsubaie @AmalAlsubaie15,Eduardo Martinez-Valdes@ mredumartinez and Deborah Falla @Deb_Falla

Contributors All authors contributed to the focus of the systematic review topic. AMA is a PhD student with DF as lead supervisor and EM-V as co-supervisor. AMA is the first reviewer for this systematic review, and MM is the second reviewer. AMA drafted the initial protocol with guidance and feedback at all stages from DF, EM-V and MM. All authors have revised and reviewed each draft of the protocol and have approved the final manuscript. DF is the guarantor.

Funding This study was funded by a PhD scholarship awarded to the first author by King Abdulaziz University in Saudi Arabia (award number: N/A).

Competing interests None declared.

Patient consent for publication Not required.

Provenance and peer review Not commissioned; externally peer reviewed.

Supplemental material This content has been supplied by the author(s). It has not been vetted by BMJ Publishing Group Limited (BMJ) and may not have been peer-reviewed. Any opinions or recommendations discussed are solely those of the author(s) and are not endorsed by BMJ. BMJ disclaims all liability and responsibility arising from any reliance placed on the content. Where the content includes any translated material, BMJ does not warrant the accuracy and reliability of the translations (including but not limited to local regulations, clinical guidelines, terminology, drug names and drug dosages), and is not responsible for any error and/or omissions arising from translation and adaptation or otherwise.

Open access This is an open access article distributed in accordance with the Creative Commons Attribution Non Commercial (CC BY-NC 4.0) license, which permits others to distribute, remix, adapt, build upon this work non-commercially, and license their derivative works on different terms, provided the original work is properly cited, appropriate credit is given, any changes made indicated, and the use is non-commercial. See: http://creativecommons.org/licenses/by-nc/4.0/.

\section{ORCID iDs}

Eduardo Martinez-Valdes http://orcid.org/0000-0002-5790-7514

Deborah Falla http://orcid.org/0000-0003-1689-6190

\section{REFERENCES}

1 Dagenais S, Caro J, Haldeman S. A systematic review of low back pain cost of illness studies in the United States and internationally. Spine J 2008;8:8-20.

2 Key J. Chapter 2 - The problem of back pain. In: Key J, ed. Back pain - a movement problem. Edinburgh: Churchill Livingstone, 2010: 3-10.

3 Mortimer M, Ahlberg G, MUSIC-Norrtälje Study Group. To seek or not to seek? Care-seeking behaviour among people with low-back pain. Scand J Public Health 2003;31:194-203.

4 Ratzon NZ, Jarus T, Catz A. The relationship between work function and low back pain history in occupationally active individuals. Disabil Rehabil 2007;29:791-6.

5 Lee J, Nussbaum MA, Kyung G. Effects of work experience on fatigue-induced biomechanical changes during repetitive asymmetric lifts/lowers. Ergonomics 2014;57:1875-85.

6 Abboud J, Nougarou F, Pagé I, et al. Trunk motor variability in patients with non-specific chronic low back pain. Eur J Appl Physiol 2014;114:2645-54.

7 Latash ML, Scholz JP, Schöner G. Motor control strategies revealed in the structure of motor variability. Exerc Sport Sci Rev 2002;30:26-31.

8 Harbourne RT, Stergiou N. Movement variability and the use of nonlinear tools: principles to guide physical therapist practice. Phys Ther 2009;89:267-82.
9 Hodges PW, Van Dieën JH, Cholewicki J. Time to reflect on the role of motor control in low back pain. Alexandria, Virginia: JOSPT, Inc. d/b/a Movement Science Media, 2019: 367-70.

10 Ebrahimi S, Kamali F, Razeghi M, et al. Comparison of the trunk-pelvis and lower extremities sagittal plane inter-segmental coordination and variability during walking in persons with and without chronic low back pain. Hum Mov Sci 2017;52:55-66.

11 Gizzi L, Röhrle O, Petzke F, et al. People with low back pain show reduced movement complexity during their most active daily tasks. Eur J Pain 2019;23:410-8.

12 Falla D, Gizzi L, Tschapek M, et al. Reduced task-induced variations in the distribution of activity across back muscle regions in individuals with low back pain. Pain 2014;155:944-53.

13 Srinivasan D, Mathiassen SE. Motor variability in occupational health and performance. Clin Biomech 2012;27:979-93.

14 Krüger M, Straube A, Eggert T. The propagation of movement variability in time: a methodological approach for discrete movements with multiple degrees of freedom. Front Comput Neurosci 2017;11:93.

15 Komar J, Seifert L, Thouvarecq R. What variability tells us about motor expertise: measurements and perspectives from a complex system approach. Movement Sport Sci 2015;89:65-77.

16 Carla C. Non-linear tools and methodological concerns measuring human movement variability: an overview. Europ J Human Movement 2014;32.

17 Hamacher D, Hamacher D, Herold F, et al. Are there differences in the dual-task walking variability of minimum toe clearance in chronic low back pain patients and healthy controls? Gait Posture 2016;49:97-101.

18 Hamacher D, Hamacher D, Schega L. A cognitive dual task affects gait variability in patients suffering from chronic low back pain. Exp Brain Res 2014;232:3509-13.

19 van den Hoorn W, Bruijn SM, Meijer OG, et al. Mechanical coupling between transverse plane pelvis and thorax rotations during gait is higher in people with low back pain. J Biomech 2012;45:342-7.

20 Wattananon P, Intawachirarat N, Cannella M, et al. Reduced instantaneous center of rotation movement in patients with low back pain. Eur Spine J 2018;27:154-62.

21 Chehrehrazi M, Sanjari MA, Mokhtarinia HR, et al. Goal equivalent manifold analysis of task performance in non-specific LBP and healthy subjects during repetitive trunk movement: effect of load, velocity, symmetry. Hum Mov Sci 2017;51:72-81.

22 Smith JA, Kulig K. Trunk-pelvis coordination during turning: a cross sectional study of young adults with and without a history of low back pain. Clin Biomech 2016;36:58-64.

23 Koch C, Hänsel F. Chronic non-specific low back pain and motor control during gait. Front Psychol 2018;9:2236.

24 Koch C, Hänsel F. Non-specific low back pain and postural control during quiet standing-a systematic review. Front Psychol 2019;10:586.

25 Le G, Chuhuai W, Haohan Z, et al. Effects of low back pain on motor performance in older people: a systematic review and meta-analysis. BMC Geriatrics 2020.

26 Furlan AD, Malmivaara A, Chou R, et al. 2015 updated method guideline for systematic reviews in the Cochrane back and neck group. Spine 2015;40:1660-73.

27 Shamseer L, Moher D, Clarke M, et al. Preferred reporting items for systematic review and meta-analysis protocols (PRISMA-P) 2015: elaboration and explanation. BMJ 2015;349:g7647.

28 Cochrane. Cochrane handbook for systematic reviews of interventions [online], 2019. Available: www.training.cochrane.org/ handbook [Accessed Jul 2019].

29 Smith V, Devane D, Begley CM, et al. Methodology in conducting a systematic review of systematic reviews of healthcare interventions. BMC Med Res Methodol 2011;11:15.

30 Dionne CE, Dunn KM, Croft PR, et al. A consensus approach toward the standardization of back pain definitions for use in prevalence studies. Spine 2008;33:95-103.

31 Preatoni E, Ferrario M, Donà G, et al. Motor variability in sports: a non-linear analysis of race walking. J Sports Sci 2010;28:1327-36.

32 Higgins JPT, Lasserson T, Chandler J. Methodological expectations of cochrane intervention reviews [online] 2016.

33 Downs SH, Black N. The feasibility of creating a checklist for the assessment of the methodological quality both of randomised and non-randomised studies of health care interventions. J Epidemiol Community Health 1998;52:377-84.

34 R R. Cochrane Consumers and Communication Review Group. Cochrane consumers and communication review group: data synthesis and analysis. Available: http://cccrg.cochrane.org [Accessed 27 Jun 2020]. 
35 Marušić MF, Fidahić M, Cepeha CM, et al. Methodological tools and sensitivity analysis for assessing quality or risk of bias used in systematic reviews published in the high-impact anesthesiology journals. BMC Med Res Methodol 2020;20:121.

36 Guyatt GH, Oxman AD, Schünemann HJ, et al. GRADE guidelines: a new series of articles in the Journal of Clinical Epidemiology. J Clin Epidemiol 2011;64:380-2.

37 Guyatt GH, Oxman AD, Vist G, et al. GRADE guidelines: 4. Rating the quality of evidence-study limitations (risk of bias). J Clin Epidemiol 2011;64:407-15.
38 Guyatt GH, Oxman AD, Kunz R, et al. GRADE guidelines: 7. Rating the quality of evidence-inconsistency. J Clin Epidemiol 2011;64:1294-302.

39 Guyatt GH, Oxman AD, Kunz R, et al. GRADE guidelines 6. Rating the quality of evidence-imprecision. J Clin Epidemio 2011;64:1283-93.

40 Guyatt GH, Oxman AD, Montori V, et al. GRADE guidelines: 5 . Rating the quality of evidence-publication bias. J Clin Epidemiol 2011;64:1277-82.

41 Balshem $\mathrm{H}$, Helfand M, Schünemann HJ, et al. GRADE guidelines: 3. Rating the quality of evidence. J Clin Epidemiol 2011;64:401-6. 